\title{
The Influence of Color Information on the Recognition of Color Diagnostic and Noncolor Diagnostic Objects
}

\author{
INÊS BRAMÃO \\ FILOMENA INÁCIO \\ LUÍS FAÍSCA \\ Universidade do Algarve \\ ALEXANDRA REIS \\ Karolinska Institutet \\ Universidade do Algarve \\ KARL MAGNUS PETERSSON \\ Max Planck Institute for Psycholinguistics \\ Universidade do Algarve
}

\begin{abstract}
In the present study, the authors explore in detail the level of visual object recognition at which perceptual color information improves the recognition of color diagnostic and noncolor diagnostic objects. To address this issue, 3 object recognition tasks with different cognitive demands were designed: (a) an object verification task; (b) a category verification task; and (c) a name verification task. The authors found that perceptual color information improved color diagnostic object recognition mainly in tasks for which access to the semantic knowledge about the object was necessary to perform the task; that is, in category and name verification. In contrast, the authors found that perceptual color information facilitates noncolor diagnostic object recognition when access to the object's structural description from long-term memory was necessary - that is, object verification. In summary, the present study shows that the role of perceptual color information in object recognition is dependent on color diagnosticity.
\end{abstract}

This work was supported by Fundação para a Ciência e Tecnologia (FCT/POCTI/ 46955/PSI/2002; FCT/PTPC/PSI-PCO/110734/2009; IBB/CBME, LA, FEDER/POCI 2010) and a PhD fellowship to Inês Bramão (FCT/SFRH/BD/27381/2006).

Address correspondence to Inês Bramão, Departamento de Psicologia, Faculdade de Ciências Humanas e Sociais, Campus de Gambelas, Universidade do Algarve, 8005-139 Faro,Portugal; mibramao@ualg.pt (e-mail). 
Keywords: color diagnosticity, color knowledge information, perceptual color information, visual object recognition, visual processing

THE VISUAL SYSTEM RECOGNIZES OBJECTS via multiple features, such as shape, color, texture, motion characteristics, and others. All of these features contribute to object recognition. The roles of these perceptual cues or properties in object recognition have been extensively investigated. For example, several studies have investigated the influence of color information on object recognition. However, some of the findings that have emerged from these studies appear inconsistent. For instance, some early studies failed to identify a role for color information in object recognition (Biederman \& Ju, 1988; Davidoff \& Ostergaard, 1988), whereas more recent investigations have reported that color input improves visual recognition, both for objects and scenes (e.g., Gegenfurtner \& Rieger, 2000; Therriault, Yaxley, \& Zwaan, 2009).

Different explanations have been proposed for these results. For instance, it has been suggested that color details improve object recognition in the following situations: when shape information is degraded (Tanaka \& Presnell, 1999), when the shape is not diagnostic for the object (Price \& Humphreys, 1989), in conditions such as low visual acuity (Wurm, Legge, Isenberg, \& Luebker, 1993) and visual object agnosia (Mapelli \& Behrmann, 1997), when the objects are from biological categories (Price \& Humphreys, 1989), and when objects are strongly associated with a color (color diagnostic objects) (Nagai \& Yokosawa, 2003; Tanaka \& Presnell, 1999). The level of color diagnosticity refers to the degree with which a particular object is associated with a specific color. For example, a strawberry, which is a color diagnostic object, is strongly associated with the color red, whereas a comb, which is a noncolor diagnostic object, is not strongly associated with any particular color. According to the color diagnosticity hypothesis (Tanaka \& Presnell, 1999), color information improves the recognition of color diagnostic objects but not noncolor diagnostic objects (see also Nagai \& Yokosawa, 2003). However, the field has not yet reached a consensus concerning these matters. Recent studies have found that perceptual color information improves object recognition independent of the semantic category and color diagnosticity (Rossion \& Pourtois, 2004; Uttl, Graf, \& Santacruz, 2006). Overall, these findings suggest that the role of perceptual color in object recognition is not well understood.

In this study, our goal was to investigate at which stage of visual processing perceptual color information modulates the recognition of color diagnostic and noncolor diagnostic objects. We hypothesized that perceptual information related to input color improves the recognition of color diagnostic and noncolor diagnostic objects at different levels of the visual recognition process. Experimental evidence has shown that perceptual color information is an important cue for activating semantic object knowledge, including the object's typical color, 
and probably other perceptual and functional properties, thus facilitating object recognition (Bramão, Faísca, Petersson, \& Reis, 2010). However, this is likely to be the case only for color diagnostic objects, because they are strongly associated with a particular color, in contrast to noncolor diagnostic objects. Moreover, there is also experimental evidence that shows that perceptual color has another role in addition to facilitating access to semantic object representations: serving as a perceptual input to the early stages of visual processing (Davidoff, Walsh, \& Wagemans, 1997; Gegenfurtner \& Rieger, 2000; Wurm, Legge, Isenberg, \& Luebker, 1993). Thus, we predict that color information also participates in the early stages of visual processing, in addition to facilitating access to the semantic representation for color diagnostic objects. However, for noncolor diagnostic objects, perceptual color is only expected to facilitate the early stages of visual processing.

To investigate this question, we constructed three different object recognition tasks for both color diagnostic and noncolor diagnostic objects: (a) an object verification task, (b) a category verification task, and (c) a name verification task. Humphreys and colleagues argued that performance of these tasks poses different challenges for the cognitive system (Humphreys, Price, \& Riddoch, 1999; Humphreys \& Riddoch, 2006; Humphreys, Riddoch, \& Quinlan, 1988; Riddoch $\&$ Humphreys, 1987). In the name verification task, participants were instructed to verify the name of visually presented objects. A number of processing stages must be completed before accessing the name representation. First, the early visual processes must encode the object shape and other perceptually available information. The encoded information must then be matched with the structural descriptions stored in long-term memory. The stored semantic and conceptual information about the object must be activated, and subsequently, the name representation is accessed. During this process, different forms of stored memory must be accessed, including knowledge about the object's shape (structural description), its functional and other meaning-related properties (semantic representation), and its name (lexical representation). In the category verification task, participants were instructed to verify the object's semantic category (natural or artifact). In contrast to name verification, category verification only depends on access to the stored structural description and the semantic representation. In the object verification task, participants were instructed to verify whether the presented object was a known object, and this only requires access to the structural description (Humphreys, Price, \& Riddoch, 1999; Humphreys \& Riddoch, 2006). By comparing the performance on these tasks, using both colored and black-and-white images, we attempted to determine the level of object processing at which color information facilitates the recognition of color diagnostic and noncolor diagnostic objects. If color information improves the recognition of color diagnostic objects both at the early visual stages and at the semantic level, then we expect to find an effect of the perceptual color for these objects when the task requires access to the structural description (i.e., in object verification). Furthermore, a larger effect 
of color information is to be expected for color diagnostic objects when the task requires access to both structural descriptions and semantic representations (i.e., in category verification). In the name verification task, we predicted color effects similar to those in the category verification task, given that no specific role of color is expected for accessing the lexical representation (i.e., the name) of an object per se.

However, if color only modulates noncolor diagnostic object recognition at the early visual processing stages, then we expect to find a perceptual color effect when the task requires access to the structural descriptions (i.e., in object verification). Moreover, we predicted that the perceptual color effect would remain constant for these objects on the remaining tasks, suggesting that only the early visual processing stages are affected by color information for these objects.

Finally, there is evidence that object recognition is faster for photographs than line drawings (Brodie, Wallace, \& Sharrat, 1991; Price \& Humphreys, 1989). Uttl and colleagues (Uttl, Graf, \& Santacruz, 2006) have argued that line drawings typically are viewed as a representation of an object class-a type-whereas photographs are viewed as a particular individual object - a token. To a certain extent, the recognition of types and tokens may recruit different perceptual and semantic processes (Uttl, Graf, \& Santacruz, 2006). In this study, we also investigated whether the color effects are the same or different for line drawings and photographs of the same objects.

\section{Method}

\section{Participants}

One hundred and forty-four Portuguese students with normal or correctedto-normal vision volunteered to participate in the experiment (Age range 18-40 years, $M=23.3, S D=4.5$; education range $12-20$ years, $M=14.7, S D=2.2$; 99 females and 45 males).

\section{Stimuli}

The initial pool of pictures consisted of 220 photos of common objects. Some photographs were selected from the Focus Multimedia CD Photo Library, some were selected from the set of Reis and colleagues (Reis, Faísca, Ingvar, \& Petersson, 2006), and some were selected via an Internet image search using the Google search engine. An independent group of 30 participants named and rated the initial set according to prototypicality, familiarity, visual ambiguity, visual complexity, and color diagnosticity. Each photo was presented for $1 \mathrm{~min}$, and the participants were asked to write down the name of the object. If they did not know the name, they were asked to choose one of the following categories: do not know name, do not know object, or tip of the tongue. Participants were 
also asked to evaluate the prototypicality of each photo "according to the degree that the presented picture represents a typical exemplar of the concept"; they were also asked to rate the degree of agreement between the presented photo and their mental image of the concept using a 5-point scale, where 1 indicated low agreement and 5 indicated high agreement. The familiarity of each photo was judged "according to how usual or unusual the object is in your realm of experience," and the participants were asked to rate the concept itself, rather than the photo, using a 5 -point rating scale ( $1=$ very unfamiliar; $5=$ very familiar $)$. The visual ambiguity of each photo was evaluated "according to how large is the group of different objects that are visually similar to he presented object" (5-point rating scale: $1=$ completely nonambiguous object; $5=$ completely ambiguous object). The visual complexity was defined as "the amount of detail or intricacy of line in the photo," and the participants were asked to rate the photo itself rather than the real-life object (5-point scale: $1=$ very low visual complexity; $5=$ very complex picture). The color diagnosticity was defined as "the degree to which the object is associated with a specific color" and was also rated on a 5-point scale ( 1 = low color diagnostic; $5=$ a high color diagnostic). These instructions are similar to those typically used in object picture rating studies (Rossion \& Pourtois, 2004; Snodgrass \& Vanderwart, 1980; Ventura, 2003).

Following the analysis of the rating scores, we selected only those photos that showed at least $80 \%$ name agreement between participants. Of these, we selected 72 photos to be used in the experiment. The objects were divided according to their color diagnosticity into a group of high-color diagnostic objects (20 from natural categories and 16 from artifact categories; Appendix A) and a group of low-color diagnostic objects (16 from natural categories and 20 from artifact categories; Appendix B). The only significant mean difference between the two groups of objects was color diagnosticity. The mean comparisons between color diagnostic and noncolor diagnostic items on the other rating variables were not significant $(p>0.20$; Table 1).

TABLE 1. Mean (and Standard Deviations) Ratings for Color Diagnosticity, Prototypicality, Familiarity, Visual Ambiguity, and Visual Complexity for Color Diagnostic and Noncolor Diagnostic Objects

\begin{tabular}{lccc}
\hline \hline Variable & $\begin{array}{c}\text { Color diagnostic } \\
\text { objects }\end{array}$ & $\begin{array}{c}\text { Noncolor } \\
\text { diagnostic objects }\end{array}$ & $\begin{array}{c}\text { Mann-Whitney } \\
\text { U test }\end{array}$ \\
\hline Color diagnosticity & $4.4(0.2)$ & $2.1(0.6)$ & $z=7.3, p<0.001$ \\
Prototypicality & $4.3(0.5)$ & $4.3(0.3)$ & $z=1.2, p=0.2$ \\
Familiarity & $4.4(0.5)$ & $4.3(0.4)$ & $z=0.3, p=0.7$ \\
Visual ambiguity & $2.4(0.8)$ & $2.2(0.7)$ & $z=0.5, p=0.6$ \\
Visual complexity & $2.6(0.6)$ & $2.7(0.6)$ & $z=-0.5, p=0.6$ \\
\hline
\end{tabular}


Each photograph was matched with a line drawing that was as similar as possible in terms of shape, size and orientation. The line drawings were selected from the picture database at the Max Planck Institute. A total of 60 of the 72 selected pictures were similar to the original Snodgrass and Vanderwart (1980) set. We used Adobe Photoshop CS2 to create four versions of each object: a color line drawing, a color photograph, a black and white line drawing and a black and white photograph (Figure 1). The color used to create the color line drawing version of the color diagnostic objects was selected by choosing the surface color of the correspondent color photograph and pasting the color onto the line drawing using the color replacement tool. To ensure that the color diagnostic objects and noncolor diagnostic objects were matched for color frequency and luminance, we applied the color of the color diagnostic objects to the noncolor diagnostic ones. Thus, the color version of a noncolor diagnostic image (both photographs and line drawings) was created by selecting the surface color of a color diagnostic object and pasting that color onto the noncolor diagnostic object using the color replacement tool. The luminance of the color-replaced image was adjusted using the brightness tool. The colored images (line drawings and photographs) of both color diagnostic and noncolor diagnostic objects were converted to grayscale using the grayscale mode, which preserves the luminance while discarding the color.

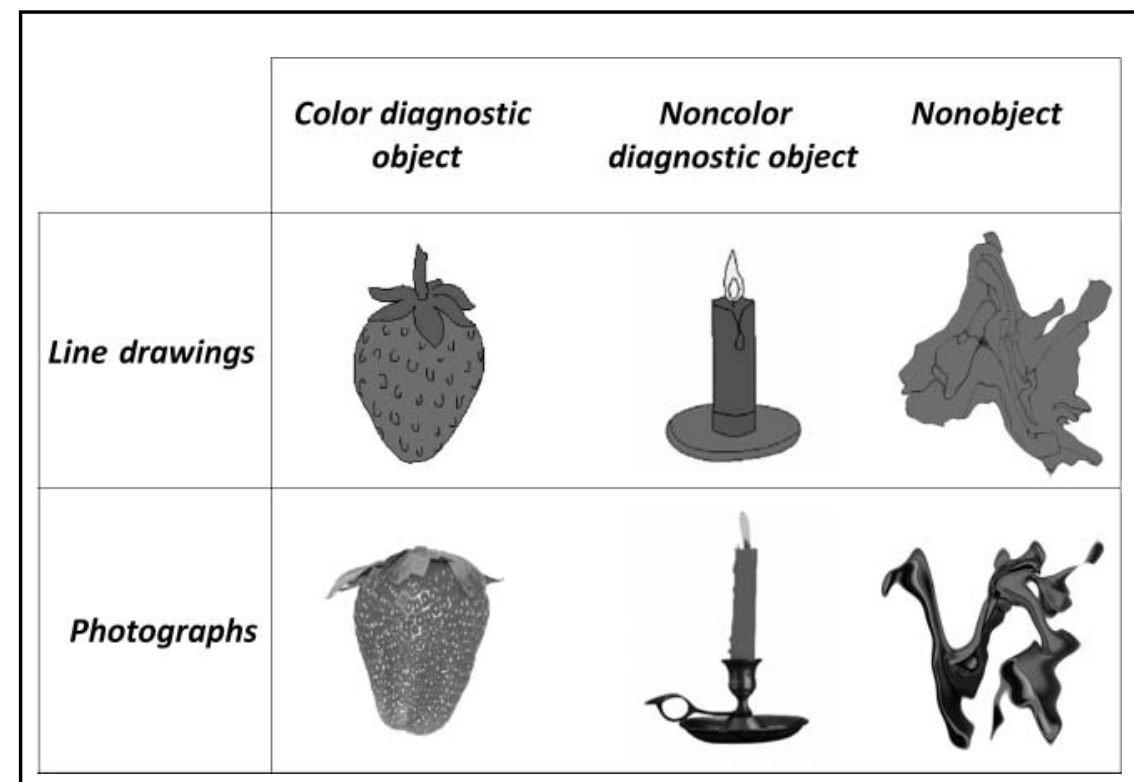

FIGURE 1. Example of the stimuli used in the experiment in the black-andwhite presentation mode. 
We did not find any difference in the luminance values between the four versions of the diagnostic and nondiagnostic items (overall $p>0.20$ ). Finally, for the object verification task, we constructed a nonobject version of each image. The nonobjects were constructed by shape deformation using the filter distort feature in Adobe Photoshop CS2. Sine waves were applied to the stimuli in a randomized fashion until it was not possible to recognize the original object shape (Figure 1).

\section{Procedures}

Three computerized verification tasks were designed, in which a picture of an object was preceded by a word: (a) an object verification task, in which before each picture, one of two possible words was presented: object or nonobject; (b) a category verification task, in which before each picture, either the word biologic or artifact was presented; and (c) a name verification task, in which, before each picture, an object name was presented. In all verification tasks, participants were asked to verify whether or not the presented picture matched the previously presented word. Each participant performed the three verification tasks (the presentation order was balanced across participants). The 72 objects and 72 nonobjects were divided into four sets of 18 objects. In each task, four different sets of objects were randomly chosen without replacement to be presented in one of the four experimental conditions, one set for each condition. In the objectverification task, four different sets of nonobjects were also randomly selected (without replacement) to be presented in one of the four experimental conditions. No participant saw an object twice in the same condition.

The category verification and the name verification tasks each comprised a total of 72 trials: half of the trials were matching trials (word and picture matched) and the other half were nonmatching trials (word and picture did not match). The nonobject verification task included 144 trials, 72 trials with objects (50-50 matching-nonmatching trials), and 72 trails with nonobjects (50-50 matching-nonmatching trials). In all the tasks, half of the trials were with color diagnostic objects (50-50 colored-black and white) and the other half were with noncolor diagnostic object presentations (50-50 colored-black and white). A specific item appeared for half of the participants associated to a matching trial and for the other half to a nonmatching trial. The Presentation 0.7 software (http://nbs.neuro-bs.com/presentation) was used to display the stimuli on a laptop Toshiba screen (Satellite A300, size: 17"; spatial resolution: $1024 \times 768$; color resolution: 24 bits) and to register the response times. Each trial started with a fixation cross displayed in the center of the screen for 1,500 ms. After the fixation cross, the word (Arial; font size 70) was presented for $1000 \mathrm{~ms}$, followed by the presentation of the object picture $(500 \times 362$ pixels $)$ for $150 \mathrm{~ms}$. The response window was $2,000 \mathrm{~ms}$, after which the next trial was presented. Participants were instructed to decide as accurately and as quickly as possible whether the word and the picture matched by pressing one of the two response keys (half of the 
participants used the right-left hand for yes-no and the other half for no-yes). Participants were allowed a break between the tasks. Before each task, participants participated in a training session with 10 trials.

\section{Results}

The results were analyzed by subject $(F 1)$ and by stimulus $(F 2)$. A minimum $F(\min F)$ was calculated from the $F 1$ and $F 2$ analyses. This approach ensured that the results were generalized over both subject and stimulus domains (Clark, 1973; Raaijmakers, 2003; Raaijmakers, Schrijnemakers, \& Gremmen, 1999). None of the main effects or interactions that failed to reach significance in the $\min F$ calculation are reported.

Overall, the participants were able to correctly verify almost all stimuli, and we focused our analysis on the verification times. We excluded the following from the analysis: verification times from incorrect responses (object verification: 3.9\%; category verification: $7.9 \%$; and name verification: $3.9 \%$ ); response times corresponding to trials where participants responded two or more times (object verification: $0.1 \%$; category verification: $0.3 \%$; and name verification: $0.3 \%$ ); response times that were greater than the response window (object verification: $0.5 \%$; category verification: $1.3 \%$; and name verification: $0.1 \%$; and no-response trials (object verification: $0.1 \%$; category verification: $0.4 \%$; and name verification: $0.3 \%$ ). The data were checked for outliers by subject and condition, and latencies outside 2.5 standard deviations from the mean for each subject and condition were also excluded from the analysis (object verification: $4.0 \%$; category verification: $3.2 \%$; and name verification: $3.9 \%$ ). We also excluded six objects that did not show any color effect (in terms of accuracy and response time) in any of the tasks (three color diagnostic objects: binoculars, cigar, and barrel; and three noncolor diagnostic objects: snake, beret, and leaf) and three participants who showed very low performance (less than $75 \%$ of correct answers in at least one of the tasks). In total, $10.1 \%$ of the response times were excluded from the analysis. A one-way analysis of variance (ANOVA) excluded object set effects $(F(3,860)=0.85 ; p=$ 0.5 ). The mean correct response times and the percentage of correct responses for each condition are given in Table 2.

Verification times were analyzed with a repeated-measures ANOVA that included the within-subject/stimulus factors of task (object verification vs. category verification vs. name verification), stimulus type (line drawings vs. photographs) and presentation mode (color vs. black and white), as well as the object color diagnosticity (color diagnostic objects vs. noncolor diagnostic objects) as a withinsubject factor in the subject analysis and a between-stimuli factor in the item analysis. The results showed a significant task effect $(F 1(2,280)=314.56, p<$ $0.001 ; F 2(2,128)=285.0, p<0.001 ; \min F(2,362)=149.5, p<0.001)$. A post-hoc comparison (Tukey HSD) for the subject analysis showed that participants were faster at name verification than object and category verification $(p<$ 
TABLE 2. Mean Response Time (and Standard Error) and Percentage of Correct Responses (and Standard Error) for Color Diagnostic and Noncolor Diagnostic Objects in Each Presentation Mode and for the Three Verification Tasks

\begin{tabular}{|c|c|c|c|c|c|c|}
\hline \multirow[b]{3}{*}{ Variable } & \multicolumn{6}{|c|}{ Verification task } \\
\hline & \multicolumn{2}{|c|}{ Object } & \multicolumn{2}{|c|}{ Category } & \multicolumn{2}{|c|}{ Name } \\
\hline & $\mathrm{RT}(S E)$ & $\%(S E)$ & $\mathrm{RT}(S E)$ & $\%(S E)$ & $\mathrm{RT}(S E)$ & $\%(S E)$ \\
\hline \multicolumn{7}{|c|}{ Color diagnostic objects } \\
\hline Color & $663(11)$ & $96(0.6)$ & $730(11)$ & $92(0.8)$ & $521(10)$ & $97(0.5)$ \\
\hline Black and white & $687(11)$ & $96(0.6)$ & $784(12)$ & $92(0.8)$ & $551(11)$ & $95(0.6)$ \\
\hline \multicolumn{7}{|c|}{ Noncolor diagnostic objects } \\
\hline Color & $652(10)$ & $96(0.7)$ & $770(13)$ & $92(1.0)$ & $532(11)$ & $96(0.6)$ \\
\hline Black and white & $686(12)$ & $97(0.6)$ & $767(12)$ & $90(1.0)$ & $542(11)$ & $96(0.6)$ \\
\hline
\end{tabular}

Note. $\mathrm{RT}=$ mean response time.

$0.001)$, and faster at object verification than category verification $(p<0.001)$. A primary effect of the presentation mode was also observed $(F 1(1,140)=67.7$, $p<0.001 ; F 2(1,64)=34.8, p<0.001 ; \min F(1,142)=22.3, p<0.001)$; participants were faster when the objects were presented in color compared to black and white. The two-way interaction between the presentation mode and the object color diagnosticity was significant $(F 1(1,140)=10.4, p<0.001 ; \mathrm{F} 2(1,64)=8.1$, $p=0.01 ; \min F(1,159)=4.5, p=0.035)$. A Tukey HSD post-hoc comparison for the subject analysis showed that when objects were presented in a color version, participants were faster at verifying color diagnostic objects than noncolor diagnostic objects $(p=0.033)$; however, when objects were presented in black and white, participants verified color diagnostic and the noncolor diagnostic objects equally quickly $(p=0.3)$. The three-way interaction between task, object-color diagnosticity and presentation mode was marginally significant $(F 1(2,280)=7.9$, $p<0.001 ; F 2(2,128)=3.2, p=0.04 ; \min F(2,236)=2.29, p=0.10$; see Table 2 and Figure 2). A Tukey HSD post-hoc comparison for the subject analysis showed that color diagnostic objects were verified equally fast in color and in black and white for the object verification task $(p=0.22)$; however, participants were faster at verifying color diagnostic objects when they were presented in color than when they were presented in black and white, both in the category verification task $(p<0.001)$ and in the name verification task $(p=0.019)$. However, the noncolor diagnostic objects were verified faster when they were presented in color than in black and white in the object verification task $(p=0.003)$. However, both in the 
(A)

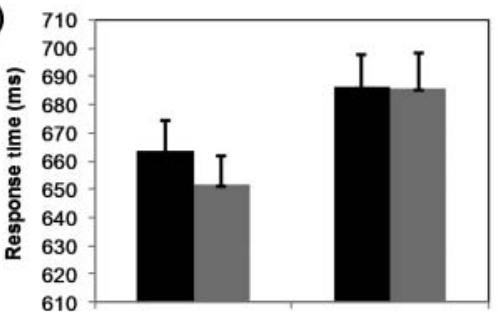

- Color diagnostic objects

= Noncolor diagnostic objects

(B)

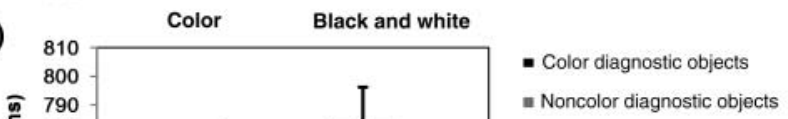

(C)

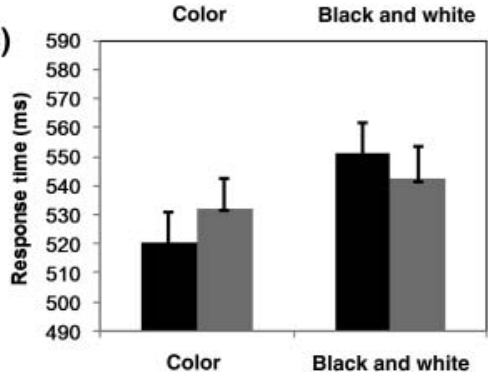

- Color diagnostic objects

= Noncolor diagnostic objects

FIGURE 2. Three-way interaction between the factors task, diagnosticity color object and presentation mode on verification times for (A) object verification task; (B) category verification task; and (C) naming verification task. Bars represent standard error.

category and in the name verification tasks, participants verified noncolor diagnostic objects equally fast when they were presented in color and in black and white $(p>0.9)$.

To avoid misinterpretation of the data and possible confusion between object color diagnosticity and semantic category effects (Nagai \& Yokosawa, 2003), we explored the color effects in both biological and artifact objects independently. The mean correct response times and the percentage of correct responses for each condition are given in Table 3. A repeated-measures ANOVA was performed that included the semantic category (biologic vs. artifacts) as a within-subject factor in 
TABLE 3. Mean Response Time (and Standard Error) and Percentage of Correct Responses (and Standard Error) for Natural and Artifact Objects in Each Presentation Mode and for the Three Verification Tasks

\begin{tabular}{|c|c|c|c|c|c|c|}
\hline \multirow[b]{3}{*}{ Variable } & \multicolumn{6}{|c|}{ Verification task } \\
\hline & \multicolumn{2}{|c|}{ Object } & \multicolumn{2}{|c|}{ Category } & \multicolumn{2}{|c|}{ Name } \\
\hline & $\mathrm{RT}(S E)$ & $\%(S E)$ & $\mathrm{RT}(S E)$ & $\%(S E)$ & $\mathrm{RT}(S E)$ & $\%(S E)$ \\
\hline \multicolumn{7}{|l|}{ Natural objects } \\
\hline Color & $664(11)$ & $96(0.6)$ & $674(11)$ & $96(0.7)$ & $521(10)$ & $97(0.5)$ \\
\hline Black and white & $685(12)$ & $95(0.6)$ & $716(11)$ & $95(0.6)$ & $552(10)$ & $95(0.7)$ \\
\hline \multicolumn{7}{|l|}{ Artifact objects } \\
\hline Color & $655(11)$ & $96(0.7)$ & $848(15)$ & $87(11)$ & $534(11)$ & $97(0.5)$ \\
\hline Black and white & $687(12)$ & $97(0.6)$ & $856(15)$ & $88(12)$ & $541(11)$ & $96(0.6)$ \\
\hline
\end{tabular}

Note. $\mathrm{RT}=$ mean response time.

the subject analysis and a between-stimuli factor in the item analysis, and task (object verification vs. category verification vs. name verification) and presentation mode (color vs. black and white) were considered a within-subject/stimulus factors. The results showed a significant semantic category effect $(F 1(1,140)=138.1$, $p<0.001 ; F 2(1,64)=102.3, p<0.001 ; \min F(1,155)=58.8, p<0.001)$; participants were quicker at responding to the biological items than to the artifact items, which was a main effect of task $(F 1(2,139)=267.7, p<0.001 ; F 2(1$, $63)=897.6, p<0.001 ; \min F(1,196)=206.2, p<0.001)$. A post-hoc comparison (Tukey HSD) for the subject analysis showed that participants were faster at name verification than object and category verification $(p<0.001)$, and object verification was performed faster than category verification $(p<0.001)$. A primary effect of presentation mode $(F 1(1,140)=73.6, p<0.001 ; F 2(1,64)=$ $32.4, p<0.001 ; \min F(1,122)=22.5, p<0.001)$ was observed; participants were faster at responding to color objects compared to black and white objects. The two-way interaction between semantic category and task was also significant $(F 1(2,139)=267.7, p<0.001 ; F 2(2,63)=897.6, p<0.001 ; \min F(1,170)=$ $52.2, p<0.001$ ); a post-hoc comparison (Tukey HSD) for the subject analysis showed that participants verified biological and artifact items equally quickly in the object and in the name verification tasks $(p>0.9)$; however, participants were faster at verifying biological than artifact objects in the category verification task $(p=0.03)$. Note that none of the interactions between category and color reached a significant level. 


\section{Discussion}

In this study, we investigated the level of visual processing at which perceptual information determined by input color facilitates the recognition process of color diagnostic and noncolor diagnostic objects. We hypothesized that perceptual color information modulates the recognition of color diagnostic and noncolor diagnostic objects at different processing stages. Specifically, we hypothesized that color information improves the recognition of color diagnostic objects both at the early visual stages and at the semantic level. In contrast, we proposed that the recognition of the noncolor diagnostic objects is only modulated at early visual processing stages; color information supports the encoding of the object shape, which facilitates access to the structural description. Consistent with our predictions, the results showed that color facilitates categorization and name verification of color diagnostic objects. This result shows that the main role of color information in the recognition of color diagnostic objects is to facilitate access to semantic object knowledge. The presence of the correct perceptual color is likely to trigger the activation of the semantic color knowledge, and this in turn, propagates through the semantic network. It was recently shown that color input is an important cue that triggers semantic knowledge related to object color, and this facilitates object recognition (Bramão, Faísca, Petersson, \& Reis, 2010). Further research is needed to explore whether additional semantic knowledge (e.g., functional properties) are also activated more quickly in the presence of the correct perceptual color information. There is some evidence that this is the case. A previous FMRI study showed that colored objects activate a neural network related to visual semantic information, which is more extensive than that for black and white objects (Bramão, Faísca, Forkstam, Reis, \& Petersson, 2010).

The color effects in the color diagnostic object recognition were not restricted to category verification; name verification was also faster in the presence of perceptual color information. However, the color effects in the task were not greater than in category verification, suggesting that color information does not contribute specifically to retrieving the object name. In other words, it appears that color input triggers the relevant semantic information, which results in faster lexical access. Consistent with this, previous evidence showed that semantic color knowledge served as a link between object shape and object name; this resulted in faster access to the name representation when activated (Davidoff, Walsh, \& Wagemans, 1997; Tanaka, Weiskopf, \& Williams, 2001). However, contrary to our predictions, we did not observe a significant effect of color information for the color diagnostic objects on the object verification task. Although, the color diagnostic objects were verified 24 milliseconds faster when the objects were presented in color (compared to black and white), this result was not significant. This nonsignificant result might suggest that the main role of perceptual color information in color diagnostic object recognition is not localized at the structural description level. Instead, our results show that the main role of color is taking effect at the semantic 
level, facilitating the activation of the semantic object network, which then results in faster lexical access. Color information may have a minor role in the recognition of color diagnostic objects at the structural description level, facilitating the extraction of the shape information and template matching in long-term memory.

In contrast, for noncolor diagnostic objects, the perceptual color effect was limited to object verification. This suggests that the role of perceptual color in noncolor diagnostic object recognition is restricted to early visual processes, including the matching of shape extraction for the structural description with the forms stored in long-term memory. We did not observe any effect of perceptual color information for the noncolor diagnostic objects on the category and name verification tasks. We noted that to succeed on these tasks, participants also had to extract and encode shape information. Nevertheless, other cognitive demands were involved; in particular, access to stored semantic information. Because color information has a limited role or no role at all in accessing the semantic information of the noncolor diagnostic objects, the effect of color on shape extraction might be masked in these tasks. This would explain why, in some studies, no color effect was observed in the naming and categorization of noncolor diagnostic objects (Nagai \& Yokosawa, 2003; Tanaka \& Presnell, 1999). Studies that showed an advantage of perceptual color information on object recognition also demonstrated that the improvement is greater for color diagnostic objects compared with noncolor diagnostic objects (Rossion \& Pourtois, 2004; Uttl, Graf, \& Santacruz, 2006).

Another interesting finding was that the observed color effect was the same for line drawings and photographs. It might seem intuitive that color would have a greater effect if it were combined with the additional surface information present in the photographs (e.g., texture and shadow), leading to faster recognition of photographs. However, this was not the case. Similar results were reported by Price and Humphreys (1989); in a naming and a categorization task, they observed that surface color and the effects of photographic detail combined subadditively, so that the combined effects were not reliably greater than either effect individually. This result suggests that both color and texture and brightness information are processed during the same time window and that both contribute independently to object recognition. The present results extend these findings. Our results show that perceptual color information is an important cue for recognizing types, as well as tokens, not only in naming and categorization tasks, but also in object decision tasks. In addition, line drawings and photographs were recognized equally fast in our study; however, previous studies have shown that photographs tend to be recognized faster than line drawings (Brodie, Wallace, \& Sharrat, 1991; Price \& Humphreys, 1989). This discrepancy might be explained by the fact that our line-drawings contained more surface details than those used in previous studies.

Previous studies have suggested that perceptual color information facilitates the recognition of natural objects but not artifacts (Price \& Humphreys, 1989). However, Nagai and Yokosawa (2003) found that regardless of the semantic 
category, perceptual color information facilitates recognition. Consistent with the latter results, our findings are not explained by the semantic category of the objects. We found that perceptual color improves the recognition of both natural objects and artifacts in similar ways. In addition, we found that participants responded faster to natural objects than to artifacts in the category verification task. The advantage of the natural objects is in agreement with studies on categoryspecific effects in healthy participants. The category-specific literature on healthy participants predicts better performance with natural objects when the viewing conditions are optimal and demands for structural differentiation are low-that is, when participants do not need to select a specific representation from long-term memory (Gale, Laws, \& Foley, 2006; Gerlach, 2009; Kiefer, 2001; Riddoch \& Humphreys, 1987).

An unexpected result in this study was the effect of task. It was thought that of the three tasks, name verification would pose the greatest cognitive challenge. Therefore, one might have expected that participants would take a longer time to respond in this task than in the other two tasks. However, this was not the case. Participants were faster at name verification compared to object verification and category verification. One possible explanation for this might be related to the labels that appeared before the stimuli in the semantic and object verification tasks: "biological" and "artifact" in category verification and "object" and "nonobject" in object verification, which are more abstract concepts than object names. Moreover, it is well-known that perceptual categorization at the basic level (e.g., dog) is faster than categorization at more superordinate (e.g., animal) or subordinate levels (e.g., poodle; Rosch, Mervis, Gray, Johnson, \& Boyes-Braem, 1976). A similar effect might play a role here: participants were faster verifying the object name (e.g., strawberry) than its superordinate category (natural vs. artifact).

Previous studies have suggested that color information is important for early visual processing (Davidoff et al., 1997; Gegenfurtner \& Rieger, 2000; Wurm et al., 1993) and/or at a semantic level, where stored semantic knowledge provides an associative link between a representation of the object shape and the object name (Davidoff et al., 1997; Tanaka et al., 2001). There is also evidence showing that stored knowledge of an object's color plays a role in that object's identification (Joseph, 1997; Joseph \& Proffitt, 1996; Mapelli \& Behrmann, 1997). Our results show that the role of perceptual color in object recognition of color diagnostic and noncolor diagnostic objects is different and depends on color diagnosticity. Perceptual color information facilitates the recognition of the color diagnostic objects at the semantic level of visual processing, while it facilitates the recognition of the noncolor diagnostic objects at the level of structural description, an earlier stage of visual processing.

\section{AUTHOR NOTES}

Inês Bramão is a PhD student in the Cognitive Neuroscience Research Group, Instituto de Biotecnologia e Bioengenharia, Universidade do Algarve. Her research is focused on 
object recognition and object naming. Filomena Inácio is a $\mathrm{PhD}$ student in the Cognitive Neuroscience Research Group, Instituto de Biotecnologia e Bioengenharia, Universidade do Algarve. Her main research interests include reading, language, memory and implicit learning. Luís Faísca is an assistant professor at the Psychology Department of Universidade do Algarve in Portugal. His main research interests include memory, reading, and language development, and quantitative analysis of behavioral data. Alexandra Reis is an associate professor at the Psychology Department of Universidade do Algarve in Portugal. Her research has focused on the cognitive neuroscience of literacy and illiteracy, reading, language, and object recognition. Karl Magnus Petersson is an assistant professor at the Psychology Department Universidade do Algarve in Portugal and a senior scientist at the Max Planck Institute for Psycholinguistics. His research is focused on the cognitive neuroscience of language, reading and writing, and learning and memory.

\section{REFERENCES}

Biederman, I., \& Ju, G. (1988). Surface versus edge-based determinants of visual recognition. Cognitive Psychology, 20, 38-64.

Bramão, I., Faísca, L., Forkstam, C., Reis, A., \& Petersson, K. M. (2010). Cortical brain regions associated with color processing: An FMRI study. Open Neuroimaging Journal, 4, 164-173.

Bramão, I., Faísca, L., Petersson, K. M., \& Reis, A. (2010). The influence of surface color information and color knowledge information in object recognition. American Journal of Psychology, 123, 459-468.

Brodie, E., Wallace, A., \& Sharrat, B. (1991). Effect of surface characteristics and style of production on naming and verification of pictorial stimuli. American Journal of Psychology, 105, 517-545.

Clark, H. (1973). The language-as-fixed-effect fallacy: A critique of language statistics in psychological research. Journal of Verbal Learning and Verbal Behaviour, 12, 335-359.

Davidoff, J., \& Ostergaard, A. (1988). The role of colour in categorial judgement. Quarterly Journal of Experimental Psychology A, 40, 533-544.

Davidoff, J., Walsh, V., \& Wagemans, J. (1997). Higher-level cortical processing of color. Acta Psychologica, 97, 1-6.

Gale, T., Laws, K., \& Foley, K. (2006). Crowded and sparse domains in object recognition: Consequences for categorization and naming. Brain and Cognition, 60, 139-145.

Gegenfurtner, K., \& Rieger, J. (2000). Sensory and cognitive contributions of color to the recognition of natural scenes. Current Biology, 10, 805-808.

Gerlach, C. (2009). Category-specificity in visual object recognition. Cognition, 111, 281-301.

Humphreys, G., Price, C., \& Riddoch, M. J. (1999). From objects to names: A cognitive neuroscience approach. Psychological Research, 62, 118-130.

Humphreys, G., \& Riddoch, M. (2006). Features, objects, action: The cognitive neuropsychology of visual object processing, 1984-2004. Cognitive Neuropsychology, 23, $156-183$.

Humphreys, G., Riddoch, M., \& Quinlan, P. (1988). Cascade processing in picture identification. Cognitive Neuropsychology, 5, 67-103.

Joseph, J. (1997). Color processing in object verification. Acta Psychologica, 97, 95-127.

Joseph, J., \& Proffitt, D. (1996). Semantic versus perceptual influences of color in object recognition. Journal of Experimental Psychology: Learning, Memory and Cognition, 22, 407-429. 
Kiefer, M. (2001). Perceptual and semantic sources of category-specifc effects: Eventrelated potentials during picture and word categorization. Memory and Cognition, 29, $100-116$.

Mapelli, D., \& Behrmann, M. (1997). The role of color in object recognition: Evidence from visual agnosia. Neurocase, 3, 237-247.

Nagai, J., \& Yokosawa, K. (2003). What regulates the surface color effect in object recognition: Color diagnosticity or category? Technical Report on Attention and Cognition, 28, 1-4.

Price, C., \& Humphreys, G. (1989). The effects of surface detail on object categorization and naming. The Quarterly Journal of Experimental Psychology, 41, 797-827.

Raaijmakers, J. (2003). A further look at the "Language-as-fixed-effect fallacy." Canadian Journal of Experimental Psychology, 57, 141-151.

Raaijmakers, J., Schrijnemakers, J., \& Gremmen, F. (1999). How to deal with "The language-as-fixed-effect fallacy": Common misconceptions and alternative solutions. Journal of Memory and Language, 41, 416-426.

Reis, A., Faísca, L., Ingvar, M., \& Petersson, K. M. (2006). Color makes a difference: Two-dimensional object naming in literate and illiterate subjects. Brain and Cognition, $60,49-54$.

Riddoch, M. J., \& Humphreys, G. (1987). Visual object processing: A cognitive neuropsychological approach. In Humphreys, G. W. \& Riddoch, M. J. (Eds.), Picture naming (pp. 107-143). London, England: Erlbaum.

Rosch, E., Mervis, C. B., Gray, W. D., Johnson, D. M., \& Boyes-Braem, P. (1976). Basic objects in natural categories. Cognitive Psychology, 8, 382-439.

Rossion, B., \& Pourtois, G. (2004). Revisiting Snodgrass and Vanderwart's object pictorial set: The role of surface detail in basic-level object recognition. Perception, 33, 217-236.

Snodgrass, J. G., \& Vanderwart, M. (1980). A standardized set of 260 pictures: norms for name agreement, image agreement, familiarity, and visual complexity. Journal of Experimental Psychology: Learning, Memory and Cognition, 6, 174-215.

Tanaka, J., \& Presnell, L. (1999). Color diagnosticity in object recognition. Perception \& Psychophysics, 61, 1140-1153.

Tanaka, J., Weiskopf, D., \& Williams, P. (2001). The role of color in high-level vision. Trends in Cognitive Sciences, 5, 211-215.

Therriault, D. J., Yaxley, R. H., \& Zwaan, R. A. (2009). The role of color diagnosticity in object recognition and representation. Cognitive Processing, 10, 335-342.

Uttl, B., Graf, P., \& Santacruz, P. (2006). Object color effects identification and repetition priming. Scandinavian Journal of Psychology, 47, 313-325.

Ventura, P. (2003). Normas para figuras do corpus de Snodgrass e Vanderwart (1980). Laboratório de Psicologia, 1, 5-19.

Wurm, L. H., Legge, G. E., Isenberg, L. M., \& Luebker, A. (1993). Color improves object recognition in normal and low vision. Journal of Experimental Psychology: Human Perception and Performance, 19, 899-911.

Original manuscript received July 9, 2010

Final version accepted October 17, 2010 
Appendix A. Color Diagnostic Objects Used in the Experiment

\begin{tabular}{llcllc}
\hline \hline Object name & Color & Diagnosticity & Object name & Color & Diagnosticity \\
\hline Banana & Yellow & 4.53 & Lemon & Yellow & 4.80 \\
Barrel & Brown & 4.07 & Lettuce & Green & 4.47 \\
Basketball & Orange & 4.40 & Light bulb & Glass & 4.33 \\
Bathing tub & Beige & 4.07 & Nail & Grey & 4.33 \\
Binoculars & Black & 4.07 & Nut & Brown & 4.60 \\
Broccoli & Green & 4.53 & Onion & Golden & 4.60 \\
Carrot & Orange & 4.73 & Orange & Orange & 4.73 \\
Cherry & Red & 4.60 & Padlock & Golden & 4.07 \\
Chick & Yellow & 4.33 & Peanut & Brown & 4.40 \\
Cigar & Brown & 4.13 & Pig & Pink & 4.33 \\
Crab & Orange & 4.53 & Pineapple & Orange & 4.73 \\
Door & Brown & 4.20 & Pipe & Brown & 4.07 \\
Dresser & Brown & 3.93 & Pumpkin & Orange & 4.47 \\
Fire extinguisher & Red & 4.40 & Strawberry & Red & 4.67 \\
Grapes & Purple & 4.13 & Table & Brown & 4.20 \\
Guitar & Brown & 4.13 & Tire & Black & 4.47 \\
Hammer & Brown & 4.07 & Tomato & Red & 4.67 \\
Kangaroo & Brown & 4.33 & Watermelon & Red & 4.53 \\
\hline
\end{tabular}

\section{Appendix B. Noncolor Diagnostic Objects Used in the Experiment}

\begin{tabular}{llcllc}
\hline \hline Object name & Color & Diagnosticity & Object name & Color & Diagnosticity \\
\hline Apple & Red & 1.93 & Funnel & Black & 1.80 \\
Bear & Brown & 2.53 & Glass & Glass & 1.40 \\
Beret & Orange & 2.53 & Glasses & Brown & 1.87 \\
Bicycle & Black & 2.47 & Glove & Pink & 1.80 \\
Book & Orange & 3.33 & Horse & Brown & 1.93 \\
Boot & Brown & 1.27 & Lamp & Golden & 2.07 \\
Bottle & Golden & 2.67 & Leaf & Green & 1.87 \\
Bowl & Purple & 2.93 & Mushroom & Brown & 1.27 \\
Bucket & Orange & 1.93 & Pen & Orange & 1.27 \\
Butterfly & Red & 1.47 & Pepper & Green & 2.93 \\
Candle & Red & 2.27 & Rabbit & Brown & 1.60 \\
Cat & Grey & 2.87 & Shirt & Red & 1.20 \\
Chicken & Beige & 1.87 & Snake & Brown & 1.80 \\
Comb & Yellow & 1.87 & Sock & Yellow & 2.40 \\
Cow & Brown & 3.27 & Tie & Yellow & 1.47 \\
Cup & Orange & 3.13 & Tulip & Red & 2.87 \\
Duck & Brown & 2.87 & Turtle & Brown & 2.67 \\
Fish & Orange & 2.93 & Watering Can & Brown & 2.13 \\
\hline
\end{tabular}

